\title{
Navigated MRI-guided liver biopsies in a closed-bore scanner: experience in 52 patients
}

\author{
Michael Moche $^{1} \cdot$ Susann Heinig $^{1} \cdot$ Nikita Garnov $^{1} \cdot$ Jochen Fuchs $^{1}$ • \\ Tim-Ole Petersen ${ }^{1}$ - Daniel Seider ${ }^{1}$ - Philipp Brandmaier ${ }^{1}$. Thomas Kahn ${ }^{1}$. \\ Harald Busse ${ }^{1}$
}

Received: 15 June 2015 / Revised: 26 August 2015 / Accepted: 28 October 2015 / Published online: 12 November 2015

(C) The Author(s) 2015. This article is published with open access at Springerlink.com

\begin{abstract}
Objectives To evaluate clinical effectiveness and diagnostic efficiency of a navigation device for MR-guided biopsies of focal liver lesions in a closed-bore scanner.

Methods In 52 patients, 55 biopsies were performed. An addon MR navigation system with optical instrument tracking was used for image guidance and biopsy device insertion outside the bore. Fast control imaging allowed visualization of the true needle position at any time. The biopsy workflow and procedure duration were recorded. Histological analysis and clinical course/outcome were used to calculate sensitivity, specificity and diagnostic accuracy.

Results Fifty-four of 55 liver biopsies were performed successfully with the system. No major and four minor complications occurred. Mean tumour size was $23 \pm 14 \mathrm{~mm}$ and the skin-to-target length ranged from 22 to $177 \mathrm{~mm}$. In 39 cases, access path was double oblique. Sensitivity, specificity and diagnostic accuracy were $88 \%, 100 \%$ and $92 \%$, respectively. The mean procedure time was $51 \pm 12 \mathrm{~min}$, whereas the puncture itself lasted $16 \pm 6 \mathrm{~min}$. On average, four control scans were taken.

Conclusions Using this navigation device, biopsies of poorly visible and difficult accessible liver lesions could be performed safely and reliably in a closed-bore MRI scanner. The system can be easily implemented in clinical routine workflow.
\end{abstract}

Michael Moche

Michael.Moche@medizin.uni-leipzig.de

1 Department of Diagnostic and Interventional Radiology, Leipzig University Hospital, Liebigstr. 20, 04103 Leipzig, Germany
Key Points

- Targeted liver biopsies could be reliably performed in a closed-bore MRI.

- The navigation system allows for image guidance outside of the scanner bore.

- Assisted MRI-guided biopsies are helpful for focal lesions with a difficult access.

- Successful integration of the method in clinical workflow was shown.

- Subsequent system installation in an existing MRI environment is feasible.

Keywords Interventional magnetic resonance imaging · Puncture biopsy $\cdot$ Image-guided biopsy $\cdot$ Stereotactic techniques $\cdot$ Liver biopsy

\section{Introduction}

Diagnostic magnetic resonance imaging (MRI) often provides superior visualization of small liver lesions that cannot be detected reliably with computed tomography (CT) or ultrasound (US) [1]. Due to the excellent soft tissue contrast, absence of ionizing radiation and longer-lasting contrast agent activity, MRI has become an advantageous alternative imaging modality for liver tumours [2] (see Fig. 1). However, as in any imaging modality, the specificity of MRI of liver lesions is not sufficiently high to preclude biopsy in most cases. While initial reports of MRI-guided percutaneous biopsies date back into the late 1980s [3-6], the implementation of these techniques into clinical practice has mostly occurred in the last decade (Table 1). One of the reasons for this delay was the later introduction of open MRI systems, which, owing to their design, provide better access to the patient compared with conventional closed-bore scanners. However, open MRI units 


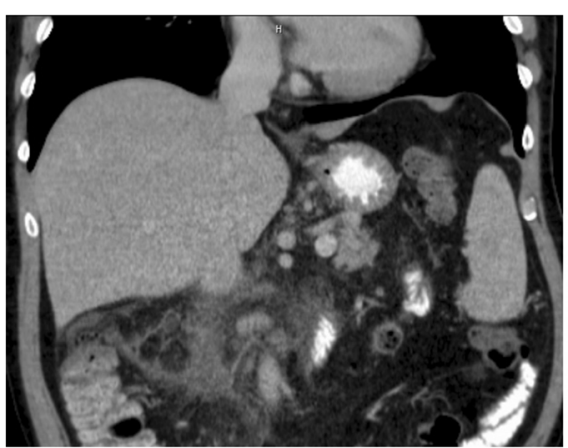

Fig. 1 A 63-year old patient with a small lesion in liver segment VII was referred for MRI-guided biopsy due to lesion invisibility on CT (left). With MRI (VIBE) the lesion (arrowhead) was clearly depicted without contrast agent administration (right). The double oblique access path was $131 \mathrm{~mm}$. With the help of the navigation device a coaxial needle is

are more costly to acquire and maintain, and provide lower image quality because of their lower magnetic field strength and inferior field homogeneity. Performing liver biopsies in conventional closed-bore high-field MRI systems has been infrequently reported, most likely because of limited patient access and, hence, the inability to guide the needle under continuous imaging. Most reports describe a time-consuming iterative approach [7-9], where after a rough assessment for an access point and orientation, the needle is stepwise guided to the lesion by multiple cycles of scanning and needle position correction outside the magnet.

An easy-to-use navigation system allowing fast and accurate determination of the skin entry point and targeting of the lesion in one step outside the bore, would simplify the above-

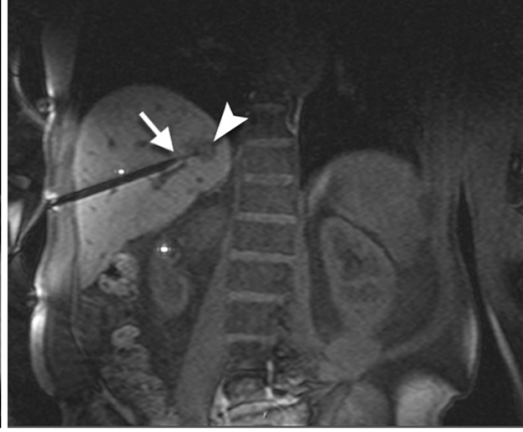

inserted before the lesion. Through the needle a $16 \mathrm{G}$ semiautomatic biopsy gun is placed. The Tru-Cut ${ }^{\mathbb{R}}$ tip of the biopsy gun is recognizable (arrow) at the border of the lesion. The histology analysis revealed a non-Hodgkin's lymphoma

mentioned approach. We report our clinical experience with liver biopsies using an add-on navigation device for a closedbore 1.5 T MRI scanner and evaluate the system's functionality, effect on workflow and diagnostic accuracy.

\section{Materials and methods}

\section{Study population}

The study was approved by the local institutional review board (University of Leipzig, register no. 344-2007) and written informed consent was obtained from each patient. Between July 2008 and February 2012, 52 patients underwent

Table 1 Literature summary of magnetic resonance-guided interventions

\begin{tabular}{|c|c|c|c|c|c|c|c|c|}
\hline Author, year & $\begin{array}{l}\text { Number of } \\
\text { interventions, } \\
\text { total/liver }\end{array}$ & $\begin{array}{l}\text { Mean } \\
\text { procedure } \\
\text { time, min }\end{array}$ & $\begin{array}{l}\text { Mean } \\
\text { intervention } \\
\text { time, min }\end{array}$ & Accuracy & $\begin{array}{l}\text { Mean skin-lesion } \\
\text { distance, } \mathrm{mm}\end{array}$ & $\begin{array}{l}\text { Mean target } \\
\text { size, } \mathrm{mm}\end{array}$ & $\begin{array}{l}\text { Minor } \\
\text { complications }\end{array}$ & MRF \\
\hline \multicolumn{9}{|l|}{ Open MRI } \\
\hline Fischbach et al., 2011 [15] & $103 / 50$ & - & 18 & - & - & 15 & 20 & yes \\
\hline Zangos et al., 2009 [24] & $322 / 126$ & - & - & 0.98 & 78 & - & - & no \\
\hline \multicolumn{9}{|l|}{ Wide-bore MRI } \\
\hline Hoffmann et al., 2012 [22] & $38 / 19$ & 134.1 & 70.6 & 0.89 & 71 & 27 & 2 & yes/no* \\
\hline Kühn et al., 2010 [18] & $47 / 44$ & 42 & 9 & 0.94 & 108 & 34 & 6 & no \\
\hline Stattaus et al., 2008 [17] & $25 / 25$ & 48 & 19 & 0.96 & 93 & 23 & 2 & yes \\
\hline \multicolumn{9}{|l|}{ Closed-bore MRI } \\
\hline Das et al., 2010 [9] & $10 / 4$ & 59.7 & $20-25$ & - & - & 25 & - & no \\
\hline Langen et al., 2002 [8] & $10 / 8$ & 48 & - & - & 70 & 62 & 1 & no \\
\hline Salomonowitz, et al., 2001 [7] & $250 / 42$ & - & 21 & 0.93 & - & - & 5 & no \\
\hline Moche et al., 2010 [19] & $6 / 1$ & - & - & - & - & - & - & no \\
\hline *Moche et al., this work & $55 / 55$ & 51 & 16 & 0.92 & 99 & 23 & 4 & no \\
\hline
\end{tabular}

$M R F$ magnetic-resonance fluoroscopy

* MRF was applied in nine cases 
MRI-guided needle biopsies of liver lesions using an add-on navigation system (iMRI Navigator ${ }^{\circledR}$, Localite, St. Augustin, Germany). We studied 30 males and 22 females with a mean age of $57.2 \pm 13.8$ years (range: $27-82$ years) and a mean body mass index (BMI) of $26.6 \pm 5.1 \mathrm{~kg} / \mathrm{m}^{2}$ (range: $17.6-40.3$ ). The patients were scheduled for MRI-guided biopsies based on the following indications: significantly improved visibility of the suspect lesion on MR images $(n=23)$ compared with $\mathrm{CT}$ (Fig. 1) and US, difficult double oblique access path $(n=39)$ or patient age under 50 years $(n=17)$.

Exclusion criteria were age under 18 years, debilitated general patient condition, uncorrectable or marginally correctable coagulation disorder, and general contraindications for MRI.

\section{Navigation technique}

Forty interventions were performed by an interventional radiologist (M.M.) with 10 years of experience; the following 15 interventions were carried out by two resident radiologists (J.F., TO.P.) with 2 years of experience under supervision. A dedicated navigation system [10] with optical tracking and automatic patient registration based on MRI-marker localization $[11,12]$ was implemented. This procedure is required only once per session but can be repeated as often as necessary. The instrument manipulation is displayed in a multiplanar virtual navigation scene on a large in-room screen. The setup of the navigation components is shown in Fig. 2.

\section{Biopsy procedure}

\section{System setup and patient preparation}

All biopsies were performed in a stable and comfortable supine patient position (Fig. 2). To improve diagnostic image quality, two 19-cm loop coils (Siemens Healthcare, Erlangen, Germany) were placed on the patients' abdomen and right side, which were used in combination with the spine coil. Two holding arms were mounted on the patient table. The holding arm opposite the access site was aligned to enable mounting of the reference marker board (RMB) as close as possible to the isocentre position of the MRIscanner. The proper line-of-sight to the camera was ensured in out-of-magnet position. The RMB is positioned in a way that it cannot be displaced by patients' breathing movements and does not interfere with sterile instruments during the intervention. A special front-end module (developed in collaboration with the authors and Invivo Germany GmbH, Schwerin, Germany), which is used to hold, adjust and lock the coaxial needle in the intended biopsy position, is attached to a holding arm on the intervention site (Fig. 2). Needle movement is visualized in the navigation display (Fig. 3) with an almost real-time refresh rate of 4 frames/s and no recognizable delay.

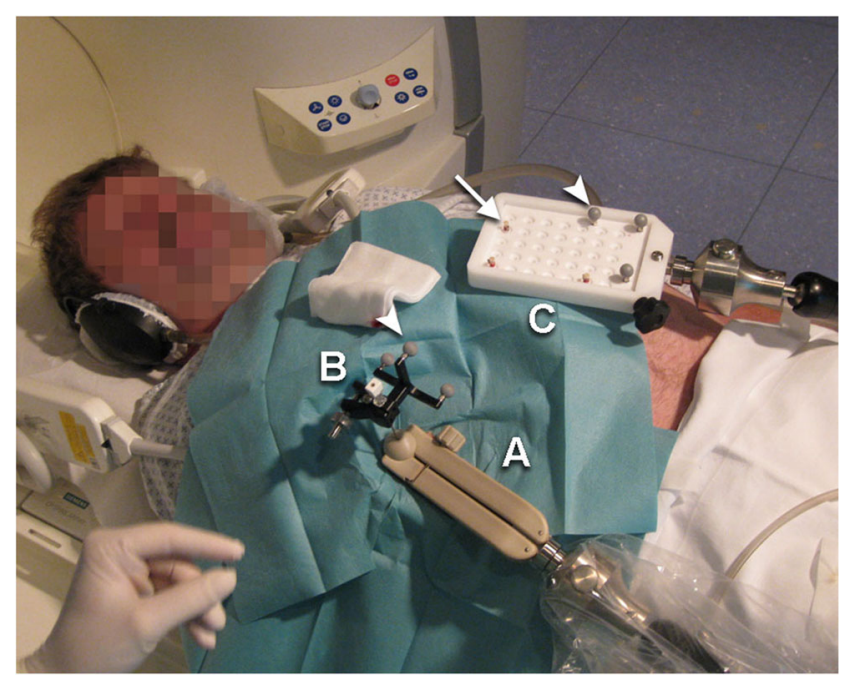

Fig. 2 Setup of the navigation components. A compact instrument tracker $(B)$ (Localite) with a set of three spherical retro-reflective optical markers (arrow head; NDI, Waterloo, ON, Canada) is attached to the base of the coaxial needle. The needle with the tracker is fixed within the frontend-module $(A)$. The main feature of the front-end module is a two-piece ball clamped in a gripping jaw into which the biopsy needle is inserted, allowing fixation in the appropriate position and orientation. Partial release of the ball enables the needle to remain stabilized in situ but allows it to follow patients' respiratory motion and with that to avoid injury to the liver. In case of emergency, the ball can quickly be detached from the front-end module, leaving the needle in situ. Optical real-time tracking of the instrument is realized through a movable 3D infrared camera (Polaris Spectra ${ }^{\circledR}$, NDI) that also couples a second reference set of the abovementioned optical markers on a reference marker board $(C)$ that remains fixed to the patient table and also features a set of MR-visible markers (arrow) that are used for registration of the navigation scene

Patient analgesia/sedation was induced by intravenous administration of 3-5 $\mathrm{mg}$ piritramide and $2-5 \mathrm{mg}$ midazolam prior to the intervention. To minimize errors caused by respiratory motion, each patient underwent a short breath-hold training before the intervention. This exercise comprised five identical breathing commands with breath-hold phases in mild inspiration, corresponding to commands given during the procedure.

\section{Imaging}

All interventions were performed in a conventional closedbore 1.5 T MRI scanner (Magnetom Symphony ${ }^{\circledR}$, Siemens) with a bore diameter of $60 \mathrm{~cm}$ and a gantry length of $150 \mathrm{~cm}$. For MR-marker localization, a short scan sequence with a low flip angle and large field of view (FOV) in three standard views was applied using the integrated body coil. This sequence aimed to provide marker projection images within a large volume $\left(300 \times 300 \times 300 \mathrm{~mm}^{3}\right)$ and minimal signal contribution from the patient, as previously described [10]. For procedure planning, transverse images were acquired with volume-interpolated breath-hold examination (VIBE), halfFourier single-shot turbo spin echo (HASTE), as well as true 

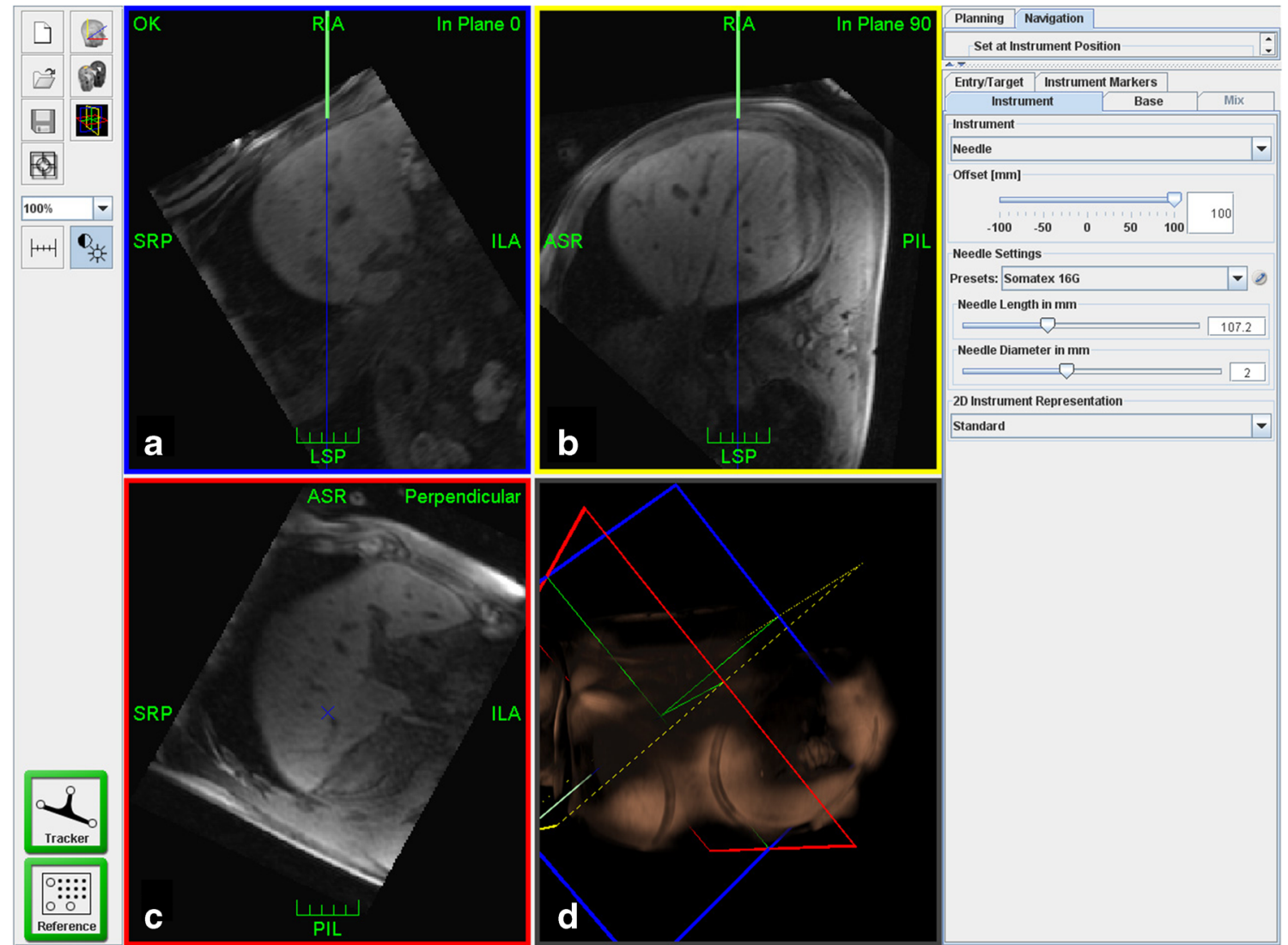

Fig. 3 Screenshot of the navigation scene. Reformatted MR images of the planning dataset are interactively shown either instrument-related inplane (a), $90^{\circ}$ in-plane (b) or perpendicular to the instrument (c). On $\mathbf{a}$ and

b the green lines indicate the needle in full length and the blue lines show its trajectory to facilitate orientation to the target (arrow head). For better orientation, a 3D reconstruction is also provided (d)

fast imaging with steady precession (TRUFI) (Table 2), using the abovementioned combination of loop and spine coils. In case the lesion was not clearly visible in the planning images, $10 \mathrm{ml}$ of the liver-specific contrast agent gadoxetate disodium (Primovist ${ }^{\circledR}$, Bayer HealthCare Pharmaceuticals, Berlin, Germany) was administered intravenously approximately $15 \mathrm{~min}$ prior to the intervention to improve negative contrast in the VIBE-sequence.

During imaging, care was taken to give the breath-hold commands as trained before to ensure an even breathing pattern and breath-hold.

The sequence with the optimum lesion visibility was used as the dataset for navigation and as standard for control imaging.

\section{Navigation procedure}

To begin navigation, marker and diagnostic images from the MRI scanner's computer were interactively sent to the navigation workstation, the software of which features modules for graphical access planning and navigation [10]. After been detected by the system, the instrument tracker was used to quickly define the puncture site with an unsterile needle.
The skin location was marked, disinfected and covered with sterile drapes. After local anaesthesia with 10-15 ml lidocaine, we made a small cutaneous incision with a scalpel. Under real-time navigation, the tip of the sterile coaxial needle (16G coaxial needle, Invivo or Somatex, SOMATEX ${ }^{\circledR}$ Medical Technologies GmbH, Teltow, Germany) attached to a sterile tracker was guided to the prepared incision site and angulated with respect to the target lesion. The access path was chosen in a way to avoid damage to pleura or adjacent organs. After giving the breath-hold command (in inspiration, as described above), the needle was rapidly inserted along the planned approach guided by the navigation images. At any time, the real needle position in situ could be verified by a control scan for which the patient table with the instrument in place was moved into the magnet. The new images could be immediately used to update the navigation scene without repeating patient registration.

\section{Sampling and post-procedure}

After successfully approaching the lesion with the coaxial needle, multiple tissue samples were obtained with the proper biopsy system (18G semiautomatic biopsy gun, Invivo). After 
Table 2 Details of diagnostic imaging sequences

\begin{tabular}{llllll}
\hline Sequence & Parameters & & & \\
\cline { 2 - 6 } & TR/TE, ms & Flip angle, & Slices, $n$ & $\begin{array}{l}\text { Thickness, } \\
\text { mm }\end{array}$ & $\begin{array}{l}\text { Acquisition } \\
\text { time }\end{array}$ \\
\hline VIBE & $3.3 / 1.3$ & 15 & 64 & 3 & $17 \mathrm{~s}$ \\
HASTE & $1100 / 120$ & 150 & 19 & 8 & $21 \mathrm{~s}$ \\
TRUFI & $4.3 / 2.2$ & 58 & 19 & 8 & $18 \mathrm{~s}$ \\
\hline
\end{tabular}

TR repetition time, TE echo time, VIBE volume-interpolated breath-hold examination, HASTE half-Fourier single-shot turbo spin echo, TRUFI true fast imaging with steady precession final retraction of the needle, control imaging with VIBE and HASTE was performed. The standard post-operation procedure in our hospital recommends the overnight stay. The patients were hospitalized over $24 \mathrm{~h}$ after the intervention to rule out potential complications, as graded by the classification of the Society of Interventional Radiology [13]. In our experience, the biopsies could be done as an outpatient treatment with $2 \mathrm{~h}$ of post-interventional monitoring and medical checkup before discharge.

\section{Evaluation}

Each procedure was documented using a standard protocol form, and the logs were evaluated retrospectively. All durations, imaging sequences, complications, contrast administration and number of samples taken were recorded. Times for individual steps were rounded to full minutes. The following procedure steps were evaluated: (i) system preparation, (ii) setup, (iii) planning, (iv) entry determination, (v) puncture preparation, (vi) intervention and (vii) control imaging (Fig. 4).

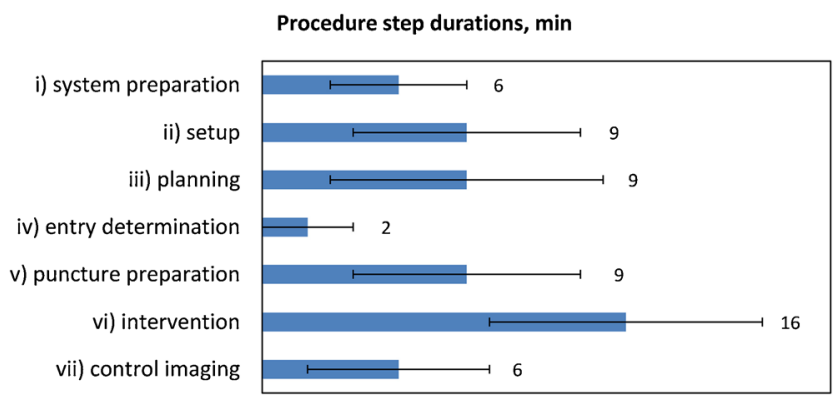

Fig. 4 Mean duration of procedural steps in minutes. (i) system preparation: placement of the infrared camera and the in-room screen, starting and booting all components; (ii) setup: patient positioning, mounting of the imaging coils, holding arms and reference marker board; (iii) planning: planning imaging, marker imaging and localization, data transfer to the navigation workstation, starting the navigation scene; (iv) entry determination: initial rough navigation with unsterile instrument tracker; (v) puncture preparation: disinfection and sterile covering; (vi) intervention: local anaesthesia, first needle insertion to sampling, final control imaging; (vii) control imaging: intermediate control imaging including patient-table travel periods (except last control), and sequence duration. Error bars indicate standard deviations
Tumour size and path length were evaluated using the scanner software. Follow-up measurements of clinical values as well as MRI or CT were performed 3 and 6 months after intervention. Statistical tests were performed using Pearson correlation analysis with a statistical significance level of $\mathrm{P}<0.05$.

The biopsy results were classified as true-negative if the samples from subsequent surgery revealed a non-malignant lesion or if the lesion was non-progressive on a subsequent MRI or CT examination for 6 months. The results were truepositive if histology confirmed malignancy. False-negative results were stated either when 6-month follow-up imaging revealed clear tumour progression or when histology after surgery was positive for malignancy.

\section{Results}

\section{Imaging and targeting}

In 52 patients, 55 liver biopsies were performed using the navigation device. During one procedure, the navigation was technically unsuccessful and the puncture was continued freehand. Most lesions were in liver segment VIII (Table 3). The VIBE sequence $(51 / 55)$ provided the best visibility of the lesions. In 37 cases, contrast agent was administrated. The mean lesion size was $23 \pm 14 \mathrm{~mm}$ (range: $5-90 \mathrm{~mm}$ ). The skin-totarget distances ranged from 22 to $177 \mathrm{~mm}$ (mean: $99 \pm$ $38 \mathrm{~mm})$. No correlation was found between tumour size $(\mathrm{r}=$ $-0.050, \mathrm{P}=0.73)$ or depth $(\mathrm{r}=0.073, \mathrm{P}=0.61)$ and intervention time. In 39 cases, the chosen access path was double oblique.

\section{Histological results}

On average, $4 \pm 1$ samples (range: $2-7$ ) were taken with each biopsy. Out of 55 biopsies, 28 revealed malignant lesions

Table 3 Lesion positions within the liver segments ${ }^{\mathrm{a}}$

\begin{tabular}{lllllllllllll}
\hline Segment & S1 & S2 & S2/3 & S3 & S4 & S5 & S5/6 & S6 & S6/7 & S7 & S7/8 & S8 \\
\hline Lesions, $n$ & 1 & 5 & 5 & 4 & 5 & 7 & 5 & 5 & 2 & 5 & 0 & 11 \\
\hline
\end{tabular}

${ }^{\text {a }}$ Liver segments are defined according to Couinaud 
(Table 4). In total, four collected samples were false-negative: one biopsy could not be reliably examined histologically because the sample was highly fragmented, but further clinical follow-up revealed a hepatocellular carcinoma. In the second false-negative case, the lesion was small $(6 \mathrm{~mm})$ and had a skin-to-target distance of $106 \mathrm{~mm}$; two further biopsies could not confirm malignancy, despite an advanced stage of the diseases (both patients died before the 6-month follow-up from cervix and pancreatic carcinoma, respectively). Two histological results suspected as malignant were excluded from the evaluation due to missing the 6-month follow-up. Overall sensitivity, specificity and accuracy were $88 \%$, $100 \%$ and $92 \%$, respectively (Table 5).

\section{Workflow}

The average duration of the procedural steps is shown in Fig. 4. The procedure time was defined as the sum of all steps except system preparation and was $51 \pm 12$ min on average (range: 24-75 $\mathrm{min}$ ). The system preparation (i) took $6 \pm 3 \mathrm{~min}$. The duration of the setup (ii) was $9 \pm 5 \mathrm{~min}$ on average. The mean duration of the planning phase (iii) was $9 \pm 6 \mathrm{~min}$. The entry point determination (iv) took $2 \pm 2 \mathrm{~min}$ (range: $1-7 \mathrm{~min}$ ). The mean intervention (vi) time including final control imaging was $16 \pm 6 \mathrm{~min}$ (range: 4-35 $\mathrm{min}$ ). The number of scans for needle position control per session ranged from 1 to 15 ( $4 \pm 1$ on average)'. The longest procedure duration was $75 \mathrm{~min}$ overall time, with $35 \mathrm{~min}$ intervention time, and 15 control scans. This biopsy was performed on a suspicious lesion in segment 1 with the longest skin-to-target distance of $177 \mathrm{~mm}$ and a difficult paracaval location (Fig. 5).

\section{Complications}

No major complications were documented for the 55 interventions. Minor complications occurred in four

Table 4 Histology results of 55 biopsies in 52 patients

\begin{tabular}{lr}
\hline Result & \\
\hline Hepatocellular carcinoma & $9(8)$ \\
Adenocarcinoma/metastasis & $9(0)$ \\
Other metastases & $10(1)$ \\
Adenoma & $3(0)$ \\
Haemangioma & $2(0)$ \\
Focal nodular hyperplasia & $4(0)$ \\
Other benign entities & $18(7)$ \\
$\quad$ (autoimmune hepatitis, inflammatory & \\
\hline
\end{tabular}

Numbers in parentheses are pathological findings with liver cirrhosis
Table 5 Diagnostic biopsy results

\begin{tabular}{lc}
\hline TP & 29 \\
TN & 20 \\
FP & 0 \\
FN & 4 \\
Sensitivity & $88 \%$ \\
Specificity & $100 \%$ \\
Accuracy & $92 \%$ \\
\hline
\end{tabular}

$T P$ true positive, $T N$ true negative, $F P$ false positive, $F N$ false negative

(7.3\%) patients. In two cases, one narrow perihepatic and one subcutaneous haematoma were detected on final control imaging. Neither showed progression on US after $24 \mathrm{~h}$. Two patients experienced moderate abdominal pain, nausea and vomiting, which regressed completely after appropriate medication which did not prolong the hospitalization period.

\section{Discussion}

For lesions that cannot be visualized reliably with US or CT, MRI can serve as an excellent imaging modality because of its high soft-tissue contrast and longer lasting contrast agent effect [14]. Nevertheless, only a few studies have reported on MRI-guided biopsies in the widely available diagnostic scanner environments. Within this study, 54 liver biopsies were successfully performed using a new targeting system to overcome limited patient access in a conventional diagnostic MRI system. The accuracy, safety and duration of the procedure were found to be acceptable for clinical routine use.

Most common MRI scanners have a bore diameter of $60 \mathrm{~cm}$ and a gantry length of $140 \mathrm{~cm}$ and above, which

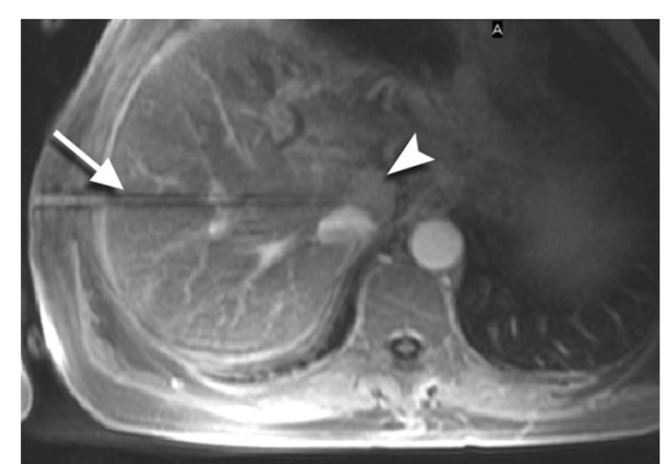

Fig. 5 An example of a difficult-to-approach paracaval lesion (arrowhead) located in segment 1 in a 72-year-old patient with liver cirrhosis. The histopathological result confirmed hepatocellular carcinoma. The image shows a maximum intensity projection reconstructed from a volume-interpolated breath-hold examination (VIBE) sequence after contrast administration with angulation along to needle pathway 
limits patient access during image acquisition. Therefore, in contrast to CT, US or open MRI, with closed-bore MRI needle insertion under continuous imaging, so-called MRfluoroscopy (MRF), is not feasible. On the other hand, MRF is especially advantageous for biopsies of uncooperative patients $[15,16]$. However, the diagnostic accuracy achieved with MRF in open or wide-bore systems does not appear higher than the accuracy reported for biopsies in closed-bore scanners without MRF (Table 1). The widebore system used by Stattaus et al. [17] enables needle insertion under MRF. However, this system and vertical open MR systems are no longer commercially available. The horizontal open scanners can have major disadvantages for procedures involving the left lobe of the liver [15]. Moreover, due to the lower magnetic field strength, and, thus, the inferior diagnostic usability, such open MRIs are becoming rarer. The wide-bore $3 \mathrm{~T}$ magnet used in previous work [18] offers a $70-\mathrm{cm}$ bore, but with a 170 -cm gantry length, the system is impractical for needle guidance under MRF.

Currently, there are only a few reports of liver biopsies performed in closed-bore high-field MRI scanners. In a small study, Das et al. [9] reported an iterative approach allowing liver biopsies to be performed within approximately $1 \mathrm{~h}$. Using a similar technique, Salomonowitz et al. [7] have performed 42 liver punctures; however, the authors did not report procedure durations. In one case, Moche et al. [19] reported application of a robotic system in a closed-bore MRI scanner for liver biopsy. Our study describes the clinical use of a navigation device in a relatively large number of liver punctures in a closed-bore MRI scanner. The system features flexibility in terms of integration into different common MRI environments, even in patients with a BMI of up to $40 \mathrm{~kg} / \mathrm{m}^{2}$, as included in this study. However, the system setup prior to the procedure requires additional time. With a mean intervention time of $51 \mathrm{~min}$ (including all steps from patient placement to final control scan), our MRI-guided procedures took longer than CT-guided procedures [20,21], even with an experienced team. However, our technique did not require significantly more time than procedures guided by MRF. In a study employing a wide-bore MRI system, even a median procedure time of approximately $134 \mathrm{~min}$ has been reported [22], which exceeds our results. Furthermore, the procedure steps have not been equally defined in different studies. Compared with scarcely described procedures using an iterative approach [9], the overall procedure times are similar to those reported here, whereas the actual intervention time appears to be shorter. It can be assumed that the time for setup and planning could be reduced by routine and sequential use of the system.

A long skin-to-target distance and small lesion size are generally indicative of the complexity of the intervention. The mean skin-to-target distance in our study was $99 \mathrm{~mm}$ and was longer than $100 \mathrm{~mm}$ in $32 \%$ of cases. These values are higher than mean distances reported by the most groups
(Table 1). This is especially noteworthy in view of the fact that iterative image-controlled placement of long needles in a closed-bore scanner is more difficult than in an open MRI system. Langen et al. [8] emphasized a long biopsy path being a limiting factor in a $60-\mathrm{cm}$ wide gantry.

The mean diameter of the lesions was $23 \mathrm{~mm}$, with $10 \%$ smaller than $10 \mathrm{~mm}$. The control imaging immediately before sampling was performed using the T1-weighted VIBE sequence, which, in conjunction with gadoxetate-based contrast agent in the majority of cases, allowed for reliable visualization of the slim Tru-Cut ${ }^{\circledR}$ needle tip in the lesion (Fig. 1). It is expected that such imaging will ensure reliable sampling. In contrast to the results of Hoffmann et al. [22], our analysis of the influence of the skin-to-target distance or lesion size on the procedure time or accuracy revealed no statistically significant correlations.

The accuracy of $92 \%$ assessed in our study is comparable with earlier reports for MRI -guided interventions [17, 18, 23, 24], as well as with the accuracy reached by US and CT guidance [25-28]. It should be noted, however, that for our study only those patients were selected who could not be biopsied under CT or US guidance. Thus, it can be assumed that this selection of difficult cases would have led to a lower accuracy when compared with a randomized cohort.

The safety of MRI-guided biopsies is emphasized by our low complication rate, which is in line with most reports [7-9, $15-19,22,23]$. There were no major complications documented, neither at our site nor in other studies, except for two cases reported from Zangos et al. [24].

Unlike neurosurgical and some other applications, the use of similar navigation techniques in organs subject to respiratory motion is controversial. Because of the lack of fluoroscopic imaging, the actual needle position cannot be controlled second-by-second. In case of patient movement or differing respiratory phases between the imaging data used for navigation and the puncture itself, the needle could be placed considerably off-target. One possibility to address this issue is motion compensation respiratory gating. This technique uses for example optical or other tracking techniques to follow abdominal wall movement and simulates the respiratory motion in the liver, applying a complex algorithm [29]. These authors concluded, however, that because of the lack of fluoroscopic imaging, motion compensation cannot be ensured with absolute certainty.

A potential drawback of this work is the fact that the employed system has not yet been approved for commercial dissemination, although the local institutional review board admitted this study. Another limitation is the lack of a control group treated with the conventional technique described by other authors [7-9]. However, due to the comparatively long mean access path in our patients and the limited space in a 60 $\mathrm{cm}$ bore, the needle should have been inserted quite deeply outside the scanner at once without any image guidance. For 
that reason the authors estimated the risk for a possible control group to be too high and decided using the system in all cases.

The presented system is less costly in comparison with dedicated interventional MRI environments. However, even with this technique liver biopsies are significantly more costintensive than CT- and especially US-guided procedures. Therefore careful selection of the patients is essential for the optimal use of this approach.

\section{Conclusion}

An approach combining brief respiratory training, navigated needle feed with automatic patient registration and immediate control imaging has proven to be successful for liver biopsies. Access to the patient outside the bore features maximum patient comfort and allows interventional radiologists to take samples with high diagnostic accuracy, even for overweight patients. Relatively short procedure durations and the flexibility of the presented add-on solution suggest integrating it into the clinical workflow in a conventional diagnostic high-field MRI environment, especially for biopsies of suspect lesions, which cannot be detected with US or CT, or are difficult to access.

\footnotetext{
Acknowledgments MM and SH contributed equally to the manuscript. The scientific guarantor of this publication is Michael Moche, MD from the Department of Diagnostic and Interventional Radiology, Leipzig University Hospital, Leipzig, Germany. The authors of this manuscript declare following relationships with companies whose products or services may be related to the subject matter of the article: Non-remunerated cooperation with Localite $\mathrm{GmbH}$ in the development of the navigation software, and non-remunerated cooperation with Invivo $\mathrm{GmbH}$ in the development of the MR-compatible needle holder.

The authors state that this work has not received any funding. No complex statistical methods were necessary for this paper. Institutional Review Board approval was obtained. Written informed consent was obtained from all patients in this study. No study subjects or cohorts have been previously reported.

Methodology: prospective, performed at one institution.
}

Open Access This article is distributed under the terms of the Creative Commons Attribution-NonCommercial 4.0 International License (http:// creativecommons.org/licenses/by-nc/4.0/), which permits any noncommercial use, distribution, and reproduction in any medium, provided you give appropriate credit to the original author(s) and the source, provide a link to the Creative Commons license, and indicate if changes were made.

\section{References}

1. Hammerstingl R, Huppertz A, Breuer J, Balzer T, Blakeborough A, Carter R et al (2008) Diagnostic efficacy of gadoxetic acid (Primovist)-enhanced MRI and spiral CT for a therapeutic strategy: comparison with intraoperative and histopathologic findings in focal liver lesions. Eur Radiol 18:457-467
2. Fowler KJ, Brown JJ, Narra VR (2011) Magnetic resonance imaging of focal liver lesions: approach to imaging diagnosis. Hepatology 54:2227-2237

3. Lufkin R, Duckwiler G, Spickler E, Teresi L, Chang M, Onik G (1988) MR body stereotaxis: an aid for MR-guided biopsies. J Comput Assist Tomogr 12:1088-1089

4. Lufkin R, Teresi L, Chiu L, Hanafee W (1988) A technique for MRguided needle placement. AJR Am J Roentgenol 151:193-196

5. Mueller PR, Stark DD, Simeone JF, Saini S, Hahn PF, Steiner E et al (1989) Clinical use of a nonferromagnetic needle for magnetic resonance-guided biopsy. Gastrointest Radiol 14:61-64

6. Schmitt R, Helmberger T, Fellner F, Obletter N (1993) The marking of nonpalpable breast tumors in MRT. Röfo 159: 484-486

7. Salomonowitz E (2001) MR imaging-guided biopsy and therapeutic intervention in a closed-configuration magnet: single-center series of 361 punctures. AJR Am J Roentgenol 177:159-163

8. Langen H-J, Kugel H, Landwehr P (2002) MR-guided core biopsies using a closed 1.0 T imager. First clinical results. Eur J Radiol 41:19-25

9. Das CJ, Goenka AH, Srivastava DN (2010) MR-guided abdominal biopsy using a 1.5-Tesla closed system: a feasibility study. Abdom Imaging 35:218-223

10. Busse H, Garnov N, Thörmer G, Zajonz D, Gründer W, Kahn T, Moche M (2010) Flexible add-on solution for MR image-guided interventions in a closed-bore scanner environment. Magn Reson Med 64:922-928

11. Garnov N, Thörmer G, Trampel R, Gründer W, Kahn T, Moche M, Busse H (2011) Suitability of miniature inductively coupled RF coils as MR-visible markers for clinical purposes. Med Phys 38: 6327-6335

12. Busse H, Trampel R, Gründer W, Moche M, Kahn T (2007) Method for automatic localization of MR-visible markers using morphological image processing and conventional pulse sequences: feasibility for image-guided procedures. J Magn Reson Imaging 26:1087-1096

13. Gupta S, Wallace MJ, Cardella JF, Kundu S, Miller DL, Rose SC et al (2010) Quality improvement guidelines for percutaneous needle biopsy. J Vasc Interv Radiol 21:969-975

14. König CW, Trübenbach J, Fritz J, Lauer UM, Claussen CD, Pereira PL (2004) Contrast enhanced MR-guided biopsy of hepatocellular carcinoma. Abdom Imaging 29:71-76

15. Fischbach F, Bunke J, Thormann M, Gaffke G, Jungnickel K, Smink J (2011) MR-guided freehand biopsy of liver lesions with fast continuous imaging using a 1.0 -T open MRI scanner: experience in 50 patients. Cardiovasc Intervent Radiol 34:188-192

16. Stattaus J, Maderwald S, Forsting M, Barkhausen J, Ladd ME (2008) MR-guided core biopsy with MR fluoroscopy using a short, wide-bore 1.5-Tesla scanner: feasibility and initial results. J Magn Reson Imaging 27:1181-1187

17. Stattaus J, Maderwald S, Baba HA, Gerken G, Barkhausen J, Forsting M et al (2008) MR-guided liver biopsy within a short, wide-bore 1.5 Tesla MR system. Eur Radiol 18:2865-2873

18. Kühn J-P, Langner S, Hegenscheid K, Evert M, Kickhefel A, Hosten $\mathrm{N}$ et al (2010) Magnetic resonance-guided upper abdominal biopsies in a high-field wide-bore 3-T MRI system: feasibility, handling, and needle artefacts. Eur Radiol 20: 2414-2421

19. Moche M, Zajonz D, Kahn T, Busse H (2010) MRI-guided procedures in various regions of the body using a robotic assistance system in a closed-bore scanner: preliminary clinical experience and limitations. J Magn Reson Imaging 31:964-974

20. Welch TJ, Sheedy PF, Johnson CD, Johnson CM, Stephens DH (1989) CT-guided biopsy: prospective analysis of 1,000 procedures. Radiology 171:493-496 
21. Silverman SG, Tuncali K, Adams DF, Nawfel RD, Zou KH, Judy PF (1999) CT fluoroscopy-guided abdominal interventions: techniques, results, and radiation exposure. Radiology 212:673-681

22. Hoffmann R, Thomas C, Rempp H, Schmidt D, Pereira PL, Claussen CD et al (2011) Performing MR-guided biopsies in clinical routine: factors that influence accuracy and procedure time. Eur Radiol 22:663-671

23. Zangos S, Kiefl D, Eichler K, Engelmann K, Heller M, Herzog C et al (2003) MR-guided biopsies of undetermined liver lesions: technique and results. Röfo 175:688-694

24. Zangos S, Müller C, Mayer F, Naguib N, Nour-Eldin N-E, Hansmann ML et al (2009) Retrospektive Analyse der 5-JahresErgebnisse von MRT-gesteuerten Biopsien im Niederfeldsystem. Röfo 181:658-663

25. Haage P, Piroth W, Staatz G, Adam G, Günther RW (1999) CTguided percutaneous biopsies for the classification of focal liver lesions: a comparison between $14 \mathrm{G}$ and $18 \mathrm{G}$ puncture biopsy needles. Röfo 171:44-48

26. Yu SC, Liew CT, Lau WY, Leung TW, Metreweli C (2001) USguided percutaneous biopsy of small $(<$ or $=1-\mathrm{cm})$ hepatic lesions. Radiology 218:195-199

27. Chojniak R, Isberner RK, Viana LM, Yu LS, Aita AA, Soares FA (2006) Computed tomography guided needle biopsy: experience from 1,300 procedures. Sao Paulo Med J 124:10-14

28. Stattaus J, Kühl H, Hauth EA, Kalkmann J, Baba HA, Forsting M (2007) Liver biopsy under guidance of multislice computed tomography: comparison of $16 \mathrm{G}$ and $18 \mathrm{G}$ biopsy needles. Radiologe 47 : 430-438

29. Maier-Hein L, Müller SA, Pianka F, Wörz S, Müller-Stich BP, Seitel A et al (2008) Respiratory motion compensation for CT-guided interventions in the liver. Comput Aided Surg $13: 125-138$ 